Document downloaded from:

http://hdl.handle.net/10251/95460

This paper must be cited as:

Climente Alarcon, V.; J. Antonino-Daviu; Riera-Guasp, M.; Vlcek, M. (2014). Induction Motor Diagnosis by Advanced Notch FIR Filters and the Wigner Ville Distribution. IEEE Transactions on Industrial Electronics. 61(8):4217-4227. doi:10.1109/TIE.2013.2286581

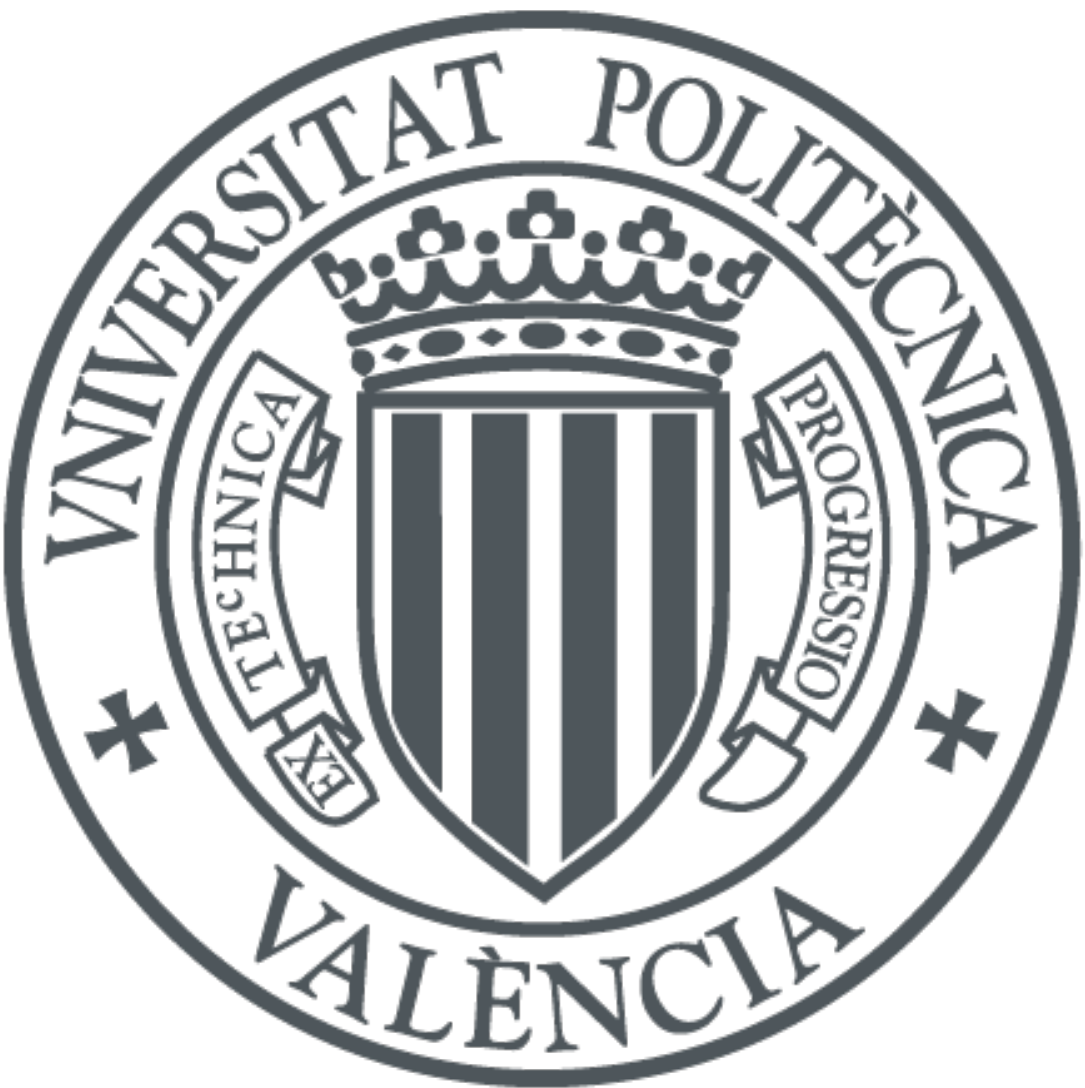

The final publication is available at

http://doi.org/ 10.1109/TIE.2013.2286581

Copyright Institute of Electrical and Electronics Engineers

Additional Information 


\title{
Induction Motor Diagnosis by Advanced Notch FIR Filters and the Wigner-Ville Distribution
}

\author{
Vicente Climente-Alarcon, Member, IEEE, Jose Antonino-Daviu, Senior Member, IEEE, Martin \\ Riera-Guasp, Senior Member IEEE and Miroslav Vlček
}

\begin{abstract}
During the last years, several time-frequency decomposition tools have been applied for the diagnosis of induction motors, for those cases in which the traditional procedures, such as the Motor Current Signature Analysis (MCSA), cannot yield the necessary response. Among them, the Cohen distributions have been widely selected to study transient and even stationary operation due to their high resolution and detailed information provided at all frequencies. Their main drawback, the cross-terms, has been tackled either modifying the distribution, or carrying out a pretreatment of the signal before computing its time-frequency decomposition. In this paper, a filtering process is proposed that uses advanced notch filters in order to remove constant frequency components present in the current of an induction motor, prior to the computation of its distribution, to study rotor asymmetries and mixed eccentricities. In transient operation of machines directly connected to the grid, this procedure effectively eliminates most of the artifacts that have prevented the use of these tools, allowing a wideband analysis and the definition of a precise quantification parameter able to follow the evolution of their state.
\end{abstract}

Index Terms-AC machines, fault diagnosis, transient, timefrequency distributions, finite impulse response filter, prognosis

\section{INTRODUCTION}

$\mathrm{T}_{\mathrm{r}}$ ransient motor current signature analysis has been recently developed for the diagnosis of induction machines in order to overcome some of the drawbacks that the analysis of stationary quantities [1]-[2] suffers in some cases, such as the difficulty of being applied to machines under unloaded condition or under unbalanced supply voltages, varying load or load torque oscillations [3]-[5].

Due to the time-varying frequency spectrum of the transient current signal, suitable time-frequency decomposition tools must be applied for its analysis. Among them, wavelet transforms (WT) [6]-[8], Hilbert-Huang transform (HHT) [9] and other Time-Frequency Distributions (TFD), as the Short Time-Fourier Transform (STFT) or the more advanced Wigner-Ville distribution (WVD), have been used.

The WVD features a relatively low computational cost and high resolution, since no windowing is applied and hence the entire signal is considered to obtain the energy at each timefrequency bin. However, its application has been hindered by the appearance in the distribution of artifacts known as cross terms. Elder and Watson already identified this problem in one of the seminal works on the study of the transient current for diagnosis of induction machines [10].
Therefore, since the early applications of quadratic representations for the detection and evaluation of fault harmonics in electrical machines, some modifications to the detection procedure have been carried out. In [11] the authors propose and experimentally validate a method for diagnosis of brushless DC motors (BLDC) based on the analysis of a phase current. In order to avoid the cross-terms, the signal is filtered before sampling by an analog adaptive filter implemented via hardware, which consisted of switched capacitors clocked by the inverter's rotor position sensor through a PLL (Phase Locking Loop). After this pretreatment (that eliminates harmonics not useful for the diagnosis) is applied, the signal is processed using a FIR filter in order to obtain the analytic signal, prior to the computation of smoothed versions of the WVD, designed to reduce the cross terms. The approach is extended in [12] to more advanced quadratic distributions, with a modified kernel for this same objective, and implemented on a DSP in order to evaluate the capacity of these computationally intensive transforms for the detection of faults in real time.

In [13] the analog adaptive filter is avoided by filtering the stator current through the Empirical Mode Decomposition (EMD) procedure with the aim of detecting short circuits in the stator of Permanent Magnet Synchronous Motors (PMSM). After that, either the Smoothed WVD or the ZhaoAtlas-Mark (ZAM) distributions are applied. In this last case, the method presents a relatively high mathematical complexity.

Urresty et Al. [14] eliminate the filtering stage in the detection of short circuits in a PMSM. Consequently, the ZAM distribution is applied directly to study high frequency harmonics. The authors argue that the distribution correctly evaluates the energy of those harmonics, taking into account that in most applications it is difficult to find stationary periods of operation for that kind of motors.

Nevertheless, all these modifications come at the expense of losing some desirable properties. For instance, the ZAM distribution, although well suited for eliminating the cross terms in signals whose components are mostly parallel in the frequency direction of the time-frequency plane, generally does not fulfill the marginals. Therefore, it does not preserve the energy in the representation, a characteristic that may be of interest in the detection of incipient faults and their evolution with the aim of estimating the remaining life of the machine (prognosis). The issue on the energy preservation was 
considered by Rosero et Al. in [15] for the detection of bearing defects on a PMSM. However, in this case the impossibility of filtering the main component, whose frequency varies in this type of machines, hinders an adequate representation of the fault harmonics by the WVD.

Furthermore, the potential of the quadratic distributions is well portrayed by Blödt et Al. [16], in spite of being a study of the stator current of an induction motor during stationary operation. In this contribution, the pseudo-WVD is used to decompose the stator current during stationary operation in order to discriminate between dynamic eccentricities and load torque oscillations and load unbalances, an especially problematic issue in the industry. Under specific conditions, both faults would cause similar sidebands around the main current component. However, the proposed procedure detects the difference in phase between the upper sideband and the lower sideband harmonic allowing a correct identification of the defect.

The preservation of the WVD's good properties whilst the cross terms are reduced is considered, for induction motors directly connected to the grid, in [17]-[18]. In those works, the use of notch filters is proposed as pretreatment to suppress the fundamental component and other constant frequency harmonics of the stator current, before computing its timefrequency distribution. This method improved the existing ones by widening the range of studied fault components evolving during the startup to higher frequencies. Such high order harmonics may provide useful information in some kinds of double faults [19], as well as under presence of low frequency load torque fluctuations. In addition, it allows the tracking and quantification of changing frequency harmonics as proposed in [20]. However, the use of infinite impulse response (IIR) filters introduces phase shifts of the current components [21], and alters the response. Additionally, these filters posses infinite step and impulse response [22], thus in the case of studying sharp transients, such as a direct startup, or noisy signals, the effect of the impulses is spread along a great time range of the distribution (Fig. 4), being sometimes its response longer than the sampling window in online applications [23].

Therefore, until now, there is still no time-frequency diagnosis method able to take advantage of the vast amount of information that those decomposition tools are capable to provide, especially that concerning the possibility of simultaneous transient tracking of high and low frequency fault components, which may be helpful in cases of difficult diagnosis, whilst avoiding the drawbacks caused by the cross terms and other interferences.

Thus, this paper presents a method to analyze stator currents of induction machines directly connected to the grid during startup transients. The main novelty of the proposed method is that it is able to portray in a single analysis both high and low variable frequency harmonics caused by rotor asymmetries and mixed eccentricities, while preserving the energy and phase of the components, thus optimizing the approach introduced in $[17,18]$. The method has a high resolution, allowing in some cases the tracking and quantization of such components even in healthy state, when they are the effect of constructive tolerances. For the outlined purpose, advanced FIR notch filters $[22,24]$ will be used in combination with the WVD to minimize the cross terms without modifying the kernel and hence preserving the good characteristics and relatively low computational cost (compared to the CWD or the ZAM) that exhibits this distribution. The results presented in this work prove the potential of the proposed transient technique to take advantage of the information provided both by the high and low frequency fault-related harmonics, increasing the reliability of the diagnostic. Furthermore, the procedure devised in this paper has been successfully applied to the study of a bar breakage during a fatigue test, where its lower computational cost allowed its application to the analysis of thousands of startups and its high precision energy assessment produced a rotor asymmetry fault indicator able to track the developing fault and hence, establish the breakage mechanism, which has especial interest for prognosis [25].

The paper is structured as follows: in Section II the behaviour of the harmonics of interest is presented. Following that, Section III introduces the current signature analysis tools and explains the detection and quantification procedure proposed. In Section IV, the experimental setup is presented, while Section V shows the experimental validation of the proposed method and finally, Section VI yields the conclusions.

\section{HARMONIC FEATURES OF INTEREST}

In this section the current components that are relevant for the diagnosis of rotor asymmetries and mixed eccentricities in induction machines are presented and their evolution during a direct startup of a motor characterized. A nomenclature for these harmonics is also introduced.

\section{A. Broken bar harmonics}

The general expression for calculating the frequencies of the components amplified by rotor bar breakages is [10]:

$$
f_{b}(s)=\left(\frac{k}{p}(1-s) \pm s\right) \cdot f \quad \frac{k}{p}=1,3,5 \ldots .
$$

where $s$ is the slip, that varies from 1 to near zero during the direct on-line startup, and $f$ the supply frequency. From this expression, two harmonics are obtained whose frequencies at steady state are slightly smaller than the frequency of the current harmonic of $k / p$ order; one of such low sidebands (LSH) evolves directly ("+" sign in (1)) and the other evolves indirectly, first reducing its frequency and then increasing it again ("-“"sign in (1)).

In addition, the pulsations in the speed of the rotor that the above mentioned components cause, trigger further descending harmonics [26] which, from multiples of the fundamental frequency, converge towards the main component as the slip decreases during the startup, to become its upper sideband harmonics (USH). Hence, for the main current component, considering both LSHs $(k / p=1$ in 
expression (1)) and USHs, it follows that:

$$
f_{b}(s)=(1 \pm 2 \cdot k \cdot s) \cdot f \quad k=1,2,3 \ldots
$$

The two main sideband harmonics often traced for the diagnosis of rotor asymmetries have the frequencies $f_{s b}$ given by (2) for $k=1$ [1], and are known simply as lower sideband harmonic (LSH) and upper sideband harmonic (USH).

Beyond these components, other authors [27] have proven the appearance of harmonic components due to the breakage located around the Principal Slot Harmonics (PSH); the frequencies of these components are given by (3) with $N_{r}$ being the number of rotor bars; $\lambda=1,2 \ldots ; v=1,3,5 \ldots[26]$.

$$
f_{P S H \pm v}(\mathrm{~s})=\left[\frac{\lambda \cdot N_{r}}{p} \cdot(1-s) \pm v\right] \cdot f
$$

Since expressions (1), (2) and (3) are directly proportional to the slip $s$, the characteristic fault-related frequencies given by them for each harmonic vary during a speed transient. In the case of a direct startup, $s$ evolves from 1 to almost 0 , yielding sharp changes in those values. This wide evolution, better tracked on a continuous time-frequency diagram, is linear with the slip thus being possible to characterize them by their frequencies when the machine is connected and at steady state. The startup evolutions of the harmonics introduced by expression (2) for $k=1,2,3$, corresponding to the Motor I, whose characteristics are shown in the Appendix, is presented in Fig. 1 (a1).

In addition, Tables I and II and Fig. 1 (a2) portray the evolution of the main high frequency lower sideband harmonics (LSHs) amplified by the asymmetry from the connection instant $(s=1)$ till steady state, assuming ideal unloaded condition $(s=0)$, according to (1). Table I characterizes the components computed using the "+" sign in (1), thus presenting a direct evolution. Table II presents the lower sideband harmonics computed using the "-" sign in (1), that is, having a negative frequency at the startup; their absolute frequency value falls progressively to zero and then increases again until reaching its steady state frequency.

TABLE I

HIGH FREQUENCY COMPONENTS AMPLIFIED BY THE ASYMMETRY ACCORDING TO (1) (DIRECT EVOLUTION).

\begin{tabular}{ccccc}
\hline & $\boldsymbol{k} / \boldsymbol{p}$ & $f \cdot\left(\frac{k}{p} \cdot(1-s)+s\right)$ & $f_{b}(s=1) H z$ & $f_{b}(s=0) H z$ \\
\hline LSH+S 150 & 3 & $f \cdot(3-2 \cdot s)$ & 50 & 150 \\
LSH+S 250 & 5 & $f \cdot(5-4 \cdot s)$ & 50 & 250 \\
LSH+S 350 & 7 & $f \cdot(7-6 \cdot s)$ & 50 & 350 \\
\hline
\end{tabular}

TABLE II

HIGH FREQUENCY COMPONENTS AMPLIFIED BY THE ASYMMETRY ACCORDING TO (1) (INDIRECT EVOLUTION).

\begin{tabular}{ccccc}
\hline & $\boldsymbol{k} / \boldsymbol{p}$ & $f \cdot\left(\frac{k}{p} \cdot(1-s)-s\right)$ & $f_{b}(s=1) H z$ & $f_{b}(s=0) H z$ \\
\hline LSH 50 & 1 & $f \cdot(1-2 \cdot s)$ & -50 & 50 \\
LSH-S 150 & 3 & $f \cdot(3-4 \cdot s)$ & -50 & 150 \\
LSH-S 250 & 5 & $f \cdot(5-6 \cdot s)$ & -50 & 250 \\
LSH-S 350 & 7 & $f \cdot(7-8 \cdot s)$ & -50 & 350 \\
\hline
\end{tabular}

Finally, Table III and Fig. 1 (a3) show the main PSHs according to (3) for the Motor I.

TABLE III

PSH COMPONENTS ACCORDING TO (3) IN HZ

\begin{tabular}{ccccc}
\hline & $\boldsymbol{\lambda}$ & $\boldsymbol{v}$ & $f_{P S H}(s=1)$ & $f_{P S H}(s=0)$ \\
\hline PSH-5 & 1 & -5 & -250 & 850 \\
PSH-3 & 1 & -3 & -150 & 900 \\
PSH-1 & 1 & -1 & -50 & 950 \\
PSH+1 & 1 & 1 & 50 & 1000 \\
PSH+3 & 1 & 3 & 150 & 1050 \\
\hline
\end{tabular}

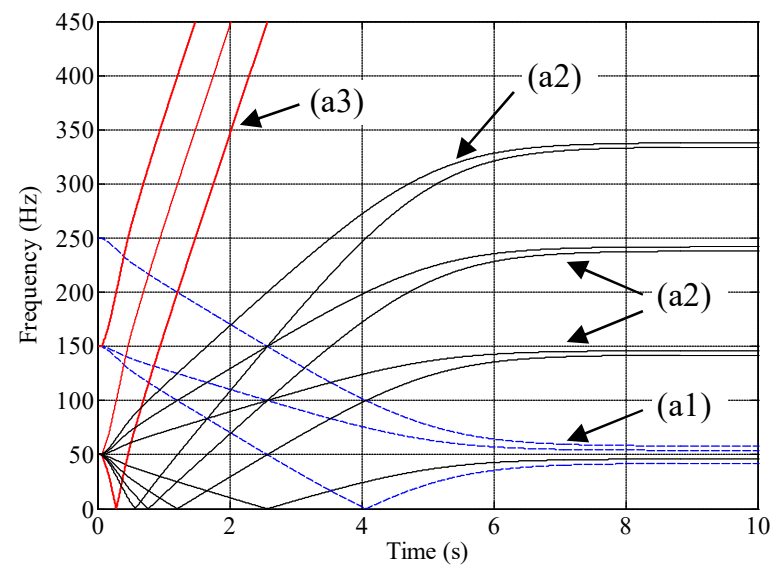

Fig. 1. Evolution of rotor asymmetry (a1, a2) and prevailing principal slot harmonics (a3) during a heavy startup for the machine tested for studying a rotor asymmetry (Appendix, Motor type I).

\section{B. Eccentricity components}

In an induction motor the eccentricity causes a non-uniform air-gap between stator and rotor. Conceptually, two main types of eccentricity exist; the static eccentricity and the dynamic eccentricity. In the case of the static eccentricity, the position of minimum air-gap remains fixed in space. On the other hand, the dynamic eccentricity appears when the rotation axis of the rotor does not overlap its geometric axis, so that the position of minimum air-gap changes with time. In practice, both types of eccentricity coexist, which is known as mixed eccentricity.

If both types of eccentricities are present, sideband components appear near the fundamental and some of its harmonics [28]. The frequencies of these components are given by (4), being $k$ an integer:

$$
f_{e c c}=\left|v \pm k \frac{1-s}{p}\right| f \quad v=1,3,5 \ldots
$$

For $v=1$ and positive frequencies, the classical expression for determining the frequency of these harmonics is obtained [29].

$$
f_{e c c}=\left(1 \pm k \frac{1-s}{p} .\right) f .
$$

Since (4) and (5) depend on the slip, as it varies from 1 to near 0 during a direct on-line startup, the components associated with the mixed eccentricity evolve in a very 
characteristic way. For the Motor type II (see characteristics in the Appendix), and $k=1$, two components diverge from the supply frequency $f(s=1)$ to reach almost $25 \mathrm{~Hz}$ and $75 \mathrm{~Hz}$ when the steady-state is reached $(s \approx 0)$ (Fig. 2, (b1)). Table IV summarizes the main components calculated from (4):

TABLE IV

ECCENTRICITY COMPONENTS ACCORDING TO (4) IN HZ

\begin{tabular}{ccccc}
\hline Component & v & $\mathbf{m}$ & $\begin{array}{c}\text { Initial frequency } \\
\text { value (connection, } \\
s=1)\end{array}$ & $\begin{array}{c}\text { Final frequency } \\
\text { value (steady-state, } \\
s=0)\end{array}$ \\
\hline EC 25 & 1 & -1 & 50 & 25 \\
EC+75 & 1 & 1 & 50 & 75 \\
EC-75 & 2 & -1 & 100 & 75 \\
EC+125 & 2 & 1 & 100 & 125 \\
EC-125 & 3 & -1 & 150 & 125 \\
EC+175 & 3 & 1 & 150 & 175 \\
\hline
\end{tabular}

The tracing of these components during the startup has been proven to be a reliable way for the detection of eccentricities [3], [30].

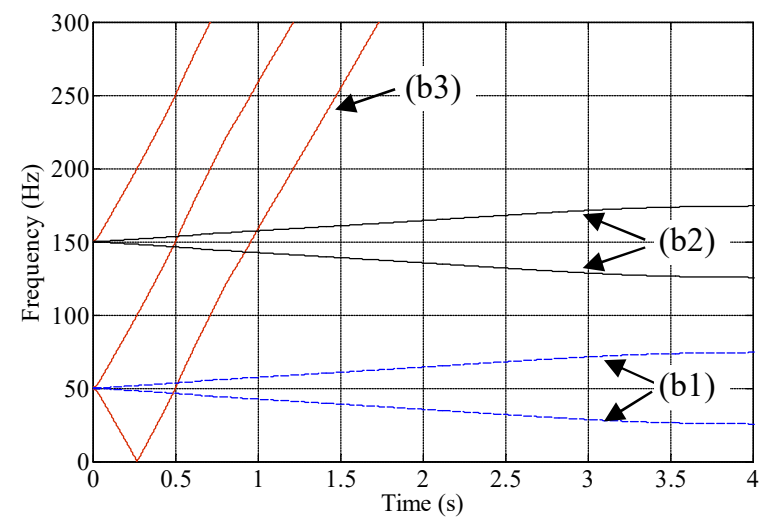

Fig. 2. Evolution of eccentricity (b1, b2) and prevailing principal slot harmonics (b3) during a heavy startup for the machine tested for studying mixed eccentricity (Appendix, Motor type II).

\section{PROPOSED ANALYSIS PROCEDURE}

In this section, the processing tools used for the diagnosis of induction motors are presented as well as the procedure followed for its implementation. The proposed approach includes a pretreatment, in which constant frequency components are eliminated using two types of optimized FIR notch filters, as well as the time-frequency distribution, applied to the residual obtained.

\section{A. Introduction to the pretreatment}

Finite impulse response filters exhibit constant group delay and limited response length which reduces the smearing of steps and impulses. In spite of these good characteristics, especially indicated for studying sharp transients, their use has been hindered by the high number of coefficients needed, compared with their IIR counterparts.

Furthermore, in the particular case of notch filters, the usual procedures utilized for the design of FIR filters, as the window algorithm or the Park-McClelland algorithm either employ numerical methods, as the former, or do not yield good results at all, as the latter.
In order to overcome these problems, in [22] is proposed a complete analytical design procedure for both maximally flat and equiripple notch filters, which allows linking by specific equations the design parameters to the characteristics of the filter (among them, the number of coefficients).

\section{B. Maximally flat-bandpass notch filters}

For the maximally flat bandpass notch filters, the authors propose a narrow bandpass FIR filter approximating its response by a polynomial. Its complementary (notch) form is:

$$
N_{p, q}(w)=1-C(1-w)^{p}(1+w)^{q}
$$

being $\mathrm{w}=\cos (\omega \mathrm{T}) \equiv \cos \lambda$, and $C$ :

$$
C=\frac{p+q}{2 q}
$$

The multiples of the roots are defined by:

$$
\begin{aligned}
& p=\left[n \cdot \sin ^{2}\left(\frac{\omega_{m} \mathrm{~T}}{2}\right)\right] \\
& q=\left[n \cdot \cos ^{2}\left(\frac{\omega_{m} \mathrm{~T}}{2}\right)\right]
\end{aligned}
$$

where the brackets denote the rounding operation and $\omega_{\mathrm{m}}$ the rejected frequency. The degree of the polynomial $n$ depends on the width of the rejection band $\Delta \lambda=\Delta \omega \mathrm{T}$ and its attenuation $a$ through the following expression:

$$
n=\frac{2 \cdot \log \left(1-10^{0.05 a}\right)}{\log \left[\cos \left(\frac{\Delta \lambda}{2}\right)\right]}
$$

The application of the analytical method provided by the authors allows the design of FIR notch filters of a moderate length, for 100 coefficients, the attenuation band $\Delta \lambda$ is 0.45 $\mathrm{rd} / \mathrm{s}$. In many practical cases, the length of the filter could be reduced neglecting the extreme terms having a value below $10^{-3}$. Beyond this attenuation band, the energy of the signal is not modified.

\section{Equiripple notch FIR filters}

In addition, the same authors propose a method for the synthesis of FIR notch filters that allows the existence of ripples in the passband [22], i.e., slightly modifying the energy signal in those non-filtered frequencies, resulting in filters with a lower number of coefficients or, equivalently, a reduction in the bandwith of the rejection band $\Delta \lambda$, feature especially interesting for obtaining a seamless time-frequency diagram.

The development of these filters is founded in the study of the Zolotarev polynomials, obtained from the solution to his first problem in approximation theory concerning polynomials that approximate constant value on a two disjoint intervals. Zolotarev developed the general solution using elliptic functions, and although Chen and Parks in 1986 [31] applied them to design FIR narrowband filters exhibiting equiripple 
behavior over the stopbands, an analytical method for their synthesis was needed in order to allow their efficient application.

This was achieved in [32] by studying the differential equations for Zolotarev polynomials defined in two disjoint intervals within $(-1,1)$, and solving, using power series, the second order linear differential equation obtained. A recursive algorithm yields the approximated polynomial.

Furthermore, the authors extend the algorithm to the expansion in the form of the Chebyshev polynomials of the Zolotarev polynomial obtained, therefore, replacing the traditional application of the FFT in order to obtain the filter coefficients from its response in frequency.

The narrow bandpass filters and their complementary notch filters are optimal in the Chebyshev sense [22], i.e, the maximum ripple in the rejection or pass bands, respectively, is minimal:

$$
\|E\|_{\infty}=\max _{-\pi \leq \lambda<\pi}|H(\lambda)-D(\lambda)|
$$

where $H(\lambda)$ is the obtained response in frequency and $D(\lambda)$ is desired response in frequency (usually flat).

These results, that allow the design of notch filters with very sharp rejection frequencies, have been used to remove the constant frequency components in the stator current and thus optimizing the method presented in [18].

\section{The Wigner-Ville distribution}

The Wigner-Ville distribution is a particular case of the Cohen class distributions. For a given signal $x(t)$ is defined by (12):

$$
W_{X}(t, \omega)=\frac{1}{2 \pi} \int_{-\infty}^{+\infty} x\left(t+\frac{\tau}{2}\right) \cdot x *\left(t-\frac{\tau}{2}\right) \cdot e^{-j \tau \omega} d \tau
$$

where $x^{*}$ denotes the conjugate of $x$. Thus, the Wigner integral is the Fourier transform, with respect to the delay variable $\tau$, of $x(t+\tau / 2) x *(t-\tau / 2)$, the instantaneous correlation. It has Hermitian symmetry in $\tau$ remaining always real [33], although it can reach negative values at some points of the distribution.

The Wigner-Ville transform presents several advantages, among them, its high resolution, which is a consequence of not using any window to limit the length of the signal before processing. In addition and contrary to other recently applied distributions [10]-[15], the Wigner-Ville distribution has the desirable property of fulfilling the marginal conditions [33], thus total signal energy can be calculated in time or in frequency using the Plancherel formula:

$$
\|x\|^{2}=\int_{-\infty}^{+\infty}|x(t)|^{2} d t=\frac{1}{2 \pi} \int_{-\infty}^{+\infty}|X(\omega)|^{2} d \omega
$$

The values $\|x(t)\|^{2}$ and $\|X(\omega)\|^{2}$ can be interpreted as energy densities in time and frequency, respectively. This enables the computation of the energy present at a given timefrequency box directly from the WVD output.

Furthermore, the WVD first conditional moment for a given time $t_{c}$ mathematically equals the instantaneous frequency
[33]:

$$
\frac{d \varphi}{d t}=\langle\omega\rangle_{t_{c}}=\frac{1}{\left|x\left(t_{c}\right)\right|^{2}} \int_{-\infty}^{+\infty} \omega W\left(t_{c}, \omega\right) d \omega
$$

Therefore, it can be computed as the average of all frequencies $\omega$ present in the time-frequency plane at a time $t_{c}$.

However, when applied to the analysis of multicomponent signals, the use of the Wigner-Ville distribution is hindered by the appearance of oscillatory interferences, among those components, at time instants and frequencies in which there should be no energy.

As a consequence, it is advisable to calculate the WVD on the analytic signal obtained from the Hilbert transform [33] of the real signal, since the Fourier transform of an analytic signal has no negative frequency components and thus, there cannot be any interference between positive and negative frequency ones.

\section{E. Implementation for fault detection}

With the aim of carrying out a fault detection, the signal processing tools presented are implemented in a three stage approach (Fig. 5):

1) Suppression of the constant components of the tested signal.

First of all, the captured current is filtered using the advanced notch filters in order to remove constant frequency components, related to the fundamental component, as well as stator winding and saturation harmonics. This procedure, which eliminates cross terms and yields a clearer representation of the results, can be applied stepwise; eliminating the main current harmonic in all cases and removing higher order ones as needed. As the studied fault component's frequencies evolve widely during a direct startup, the problems seen in the application of notch filters to the study of rotor asymmetries in stationary operation, that is, the attenuation of the sidebands [23], have no influence in this case. In addition, for certain sampling rates, comb filters based in the same approach, as presented in [24] can be used to filter the constant frequency harmonics greatly reducing the number of filter coefficients.

When the study is focused on a specific time-frequency box, the signal is also filtered by means high, low or bandpass FIR filters. Further, down sampling eases the computational requirements.

2) Computation of the analytic signal

In order to avoid the cross terms produced as a result of positive and negative frequency components interfering, the analytic signal is obtained from the filtered data by means of the Hilbert transform [33], in the second step of the data processing.

\section{3) Application of the WVD}

Finally, the time range is selected and the WVD of the resulting signal is calculated using the TFTB Toolbox developed by CNRS (France) and Rice University (USA) for MATLAB [34]. 


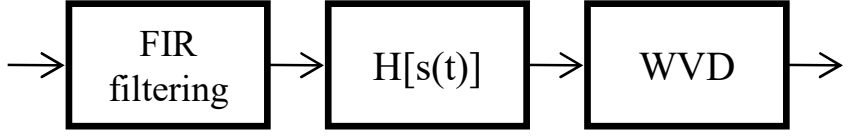

Fig. 3. Proposed signal analysis approach. A FIR filtering pretreatment removes constant frequency harmonics and limits a time-frequency box, if necessary. The analytical signal of the residual is obtained in the second step and finally the WVD is computed.

The advantages of the combination of the FIR filters and the Wigner-Ville distribution are well portrayed in Fig. 4. IIR filters, as used in $[17,18]$ cause the smearing of impulses (Fig. 4 (a)) and a consequent increase of cross terms in the timefrequency distribution, whilst FIR filters cannot extend the influence of any sharp transient (including the ones caused by noise) beyond their own coefficient's length (Fig. 4 (b))

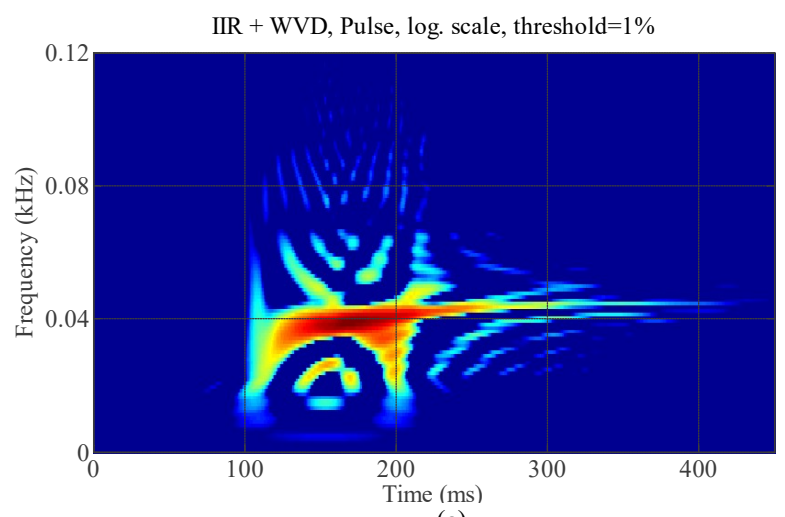

(a)

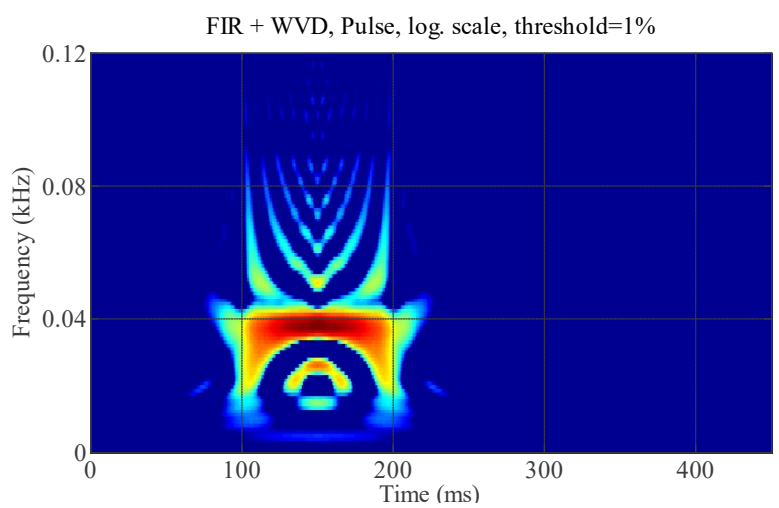

(b)

Fig. 4. Application of the detection approach to a $100 \mathrm{~ms}, 38 \mathrm{~Hz}$ pulse, (a) using IIR filters as pretreatment [17, 18], (b) using equiripple FIR filters proposed in this work. The smearing effect and associated cross terms on the time-frequency diagram due to the infinite impulse response time are clear in the first case.

\section{F. Implementation for Quantification}

The quantification procedure proposed in this paper, derived from [35], consists of obtaining, following the steps 1 to 3 presented in the previous point, a suitable time-frequency box in which a fault component prevails. In addition:

4) Using (14), the instantaneous frequency for each time instant of that time-frequency box is computed. The result oscillates sharply, thus it is smoothed, firstly discarding the values above and below the frequency limits of the considered time-frequency box and then using a low pass filter, whose pole is adjusted in order to avoid the influence of noise in the evolution of the instantaneous frequency.

5) The result follows the evolution of the prevailing fault harmonic, and hence its energy $e_{i f, b w}$, which reflects the degree of the fault, can be computed by integrating the value of the WVD on a narrow band, whose width $b w$ is determined empirically, centred for each time in that instantaneous frequency if (15):

$$
e_{i f, a}=\sum_{t} \sum_{f=i f-b w / 2}^{i f+b w / 2} W V D\left(f, t_{c}\right)
$$

where if is the instantaneous frequency in each time interval $t_{c}$ of the time-frequency box considered, $b w$ is the bandwidth and WVD is the Wigner-Ville distribution computed for that time-frequency box.

The ratio of this value over the energy in the original current waveform for the same time limits yields a quantification parameter:

$$
\gamma_{W, \chi}=10 \cdot \log \left[\frac{2 \sum_{t} i^{2}}{e_{i f, b w}}\right]
$$

where, $\chi$ is the name of the evaluated component, $i$ is the instantaneous value of the current, whose energy is integrated in the same time range and sampling frequency as in (15). Since the computation of the analytical signal doubles the energy of the original real one, the value of the numerator in (16) is increased twofold.

Thus, this quantification procedure presents the advantage of integrating the energy in a band which is centred on the fault component, minimizing the contributions of noise and other small amplitude fault harmonics, always present in the startup current due to manufacturing tolerances, therefore yielding a more stable fault indicator as compared to the techniques such as the one proposed in [36] and applied in [25], based on the Discrete Wavelet transform, which evaluates the energy present in a complete time-frequency box. Furthermore, the use of FIR filters allow a lower distortion of the energy of the detected harmonics, from an attenuation of $60 \%$ in the bands of $0-40 \mathrm{~Hz}$ and $210-240 \mathrm{~Hz}$ in $[17,18]$ to $25 \%$ using the equiripple approach.

\section{EXPERIMENTAL SETUP}

In order to validate the proposed approach, tests were carried on two types of commercial motors. Their characteristics are shown in the Appendix.

\section{A. Rotor asymmetry}

For the case of the rotor asymmetry, two rotors, the first being healthy and the second having a rotor bar drilled at its junction with the short circuit ring (Fig. 5), were successively assembled in the same stator of a one pole pair motor. 


\section{B. Eccentricity}

In the case of the eccentricity, the original shaft position was modified by replacing the end ball bearings of the motor (see Fig. 6(a)) by roller bearings (Fig. 6(d)) with greater inner diameter and smaller outer diameter. The clearance gained allowed accommodating two precision eccentric machined steel sockets (Fig. 6(b) and 6(c)). The cylindrical surfaces of both sockets were eccentric, $0.15 \mathrm{~mm}$ in the case of the outer ring $\mathrm{b}$, and $0.25 \mathrm{~mm}$ in the case of the inner ring c. Fig. 6(e) shows the new assembly mounted on the shaft, obtaining in this way a rotor having a $30 \%$ static eccentricity and a $50 \%$ dynamic eccentricity.

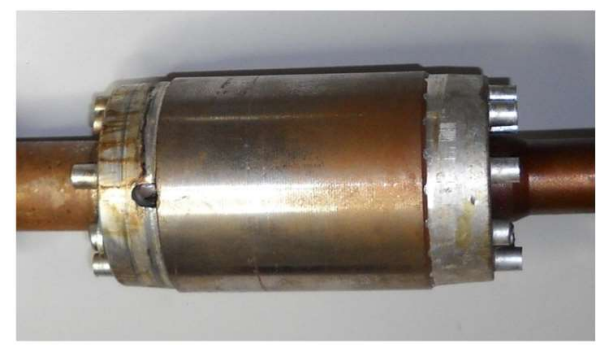

Fig. 5. Rotor featuring a broken bar.

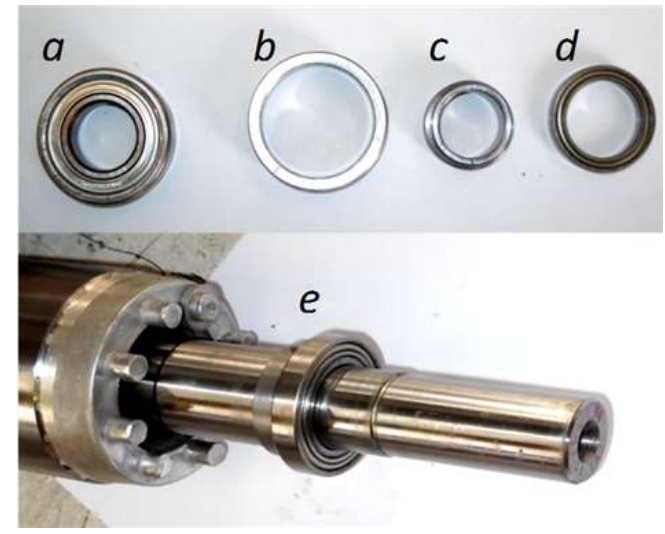

Fig. 6. Rotor of the eccentric motor unit. Top, from left to right: a) original bearing, b) external and c) internal eccentric rings, and d) new bearing. Bottom: e) mounted unit on the shaft.

\section{Test stand}

For loading purposes, a DC machine, with an inertia of 0.11 $\mathrm{kg} \cdot \mathrm{m}^{2}$ was used (Fig. 7). This relatively large inertia allowed, for the motors used in the validation of the approach, startups of up to seven seconds for the case of the one pole pair machine, and 3 seconds for the two pole pair one.

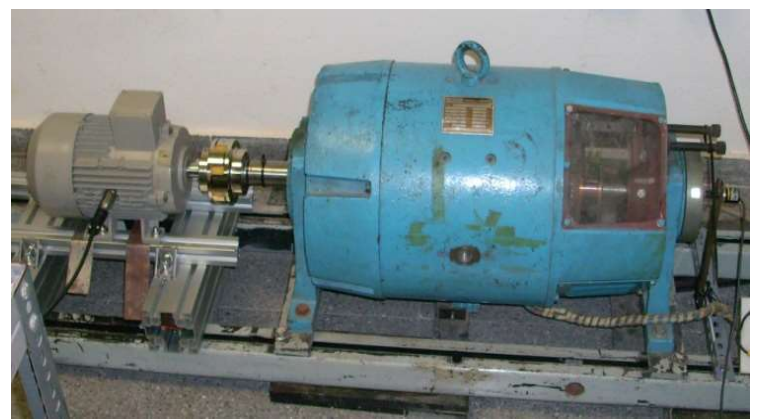

Fig. 7. Experimental test bench. Induction motor and load.

For the acquisition of the currents used as diagnostic signal; a $15 / 5$, class 0.5 current transformer and a $1 \mathrm{~A}, 60 \mathrm{mV}$ shunt were utilized; the resulting voltage signal was captured by means of a digital oscilloscope with a sampling frequency $f_{s}=$ $5000 \mathrm{samples} / \mathrm{s}$ and 16 bits of resolution, and finally transferred to a PC for the analyses in MATLAB.

The signals obtained following the aforementioned procedures underwent two analysis methods; the first one yields a wideband time-frequency diagram to provide an insight of the evolution of several fault harmonics, which enhances the possibility of correctly detecting the presence of the fault. The seamless evolution of the fault harmonics provided by the FIR filter allows an easier implementation of image recognition techniques to automatize this task. The second procedure focuses on a determined time-frequency box in order to perform a quantification of the energy contained in one of such fault harmonics, with the aim of determining the severity of the failure.

\section{RESUlTS AND DISCUSSION}

In this section, the results obtained from the application of the proposed approach to the induction motors are presented.

\section{A. Connection instant}

The analysis of the connection instant of an induction motor directly fed from the grid presents great difficulties due to the influence of the sharp initial impulse linked to the electromagnetic transient.

However, the application of the maximally flat bandpass notch filters introduced in Section III exhibit low smearing of the initial impulse. Its effect is spread only to a number of samples equal to the length of the filter, and even in this case most of the energy is concentrated in the connection instant, allowing tracking some PSHs from their origin, as it is seen in Fig 8 , in which two filters of these characteristics have eliminated the main harmonic and the $250 \mathrm{~Hz}$ winding harmonic for studying the startup of a healthy Motor type I. The PSH +3 is clearly identified, having a frequency at connection of $150 \mathrm{~Hz}$ (c1), as exposed in Table III, and directly evolving towards high frequencies. The evolution of the also prevailing PSH-1 is detected since the 100th millisecond after connection (c2), as it reduces its frequency towards zero and then increases it again towards its steady state around $1 \mathrm{kHz}$.

\section{B. High order harmonics in healthy state}

In order to test the sensitiveness of the method, the second half of the startup of a healthy motor is studied. In this case, a full pretreatment has been used, and the equiripple notch filters, having a narrower attenuation band than the flat band pass ones for the same number of coefficients, have been employed to eliminate the main current component and the $150 \mathrm{~Hz}$ and $250 \mathrm{~Hz}$ constant harmonics, since in this case, the time-frequency boxes studied are far away the connection impulse. Furthermore, the result has been band pass filtered 
and down sampled, leaving just the frequencies where the high frequency harmonics caused by a rotor asymmetry evolve (70$300 \mathrm{~Hz}$ ). The resulting diagram is shown in Fig. 9. A similar result using fewer filter coefficients can be obtained resampling the signal to $800 \mathrm{~Hz}$ and using the comb filters proposed in [24], due to the symmetric separation of the constant frequency harmonics to be eliminated at this sampling rate.

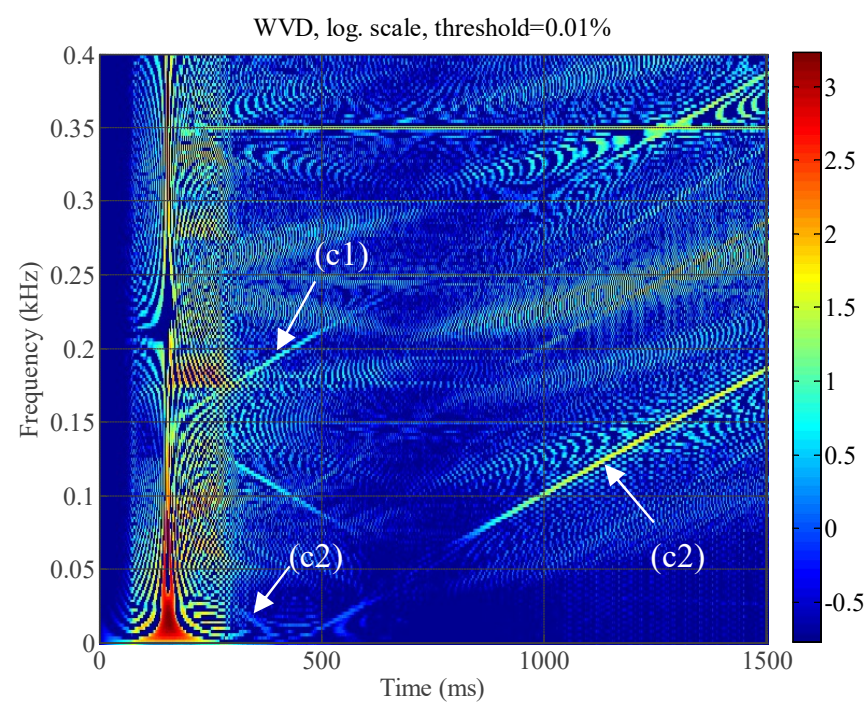

Fig. 8. Connection instant and first second of a direct startup, healthy state

In this case, the evolution of the $\mathrm{PSH}$, now reaching higher frequencies, is seen at the beginning of the diagram (d1). However, even for a healthy machine, high order harmonics due to a rotor asymmetry can be appreciated (d2), as they evolve to become low sidebands of the $250 \mathrm{~Hz}$ winding harmonic. A USH of the main current component (d3) is also shown, as well as part of the filtered LSH 50 (d4). All of them are caused by the inherent constructive tolerances of the machine. This diagram can be compared to the theoretical of Fig. 1

WVD healthy, log. scale, threshold $=0.1 \%$

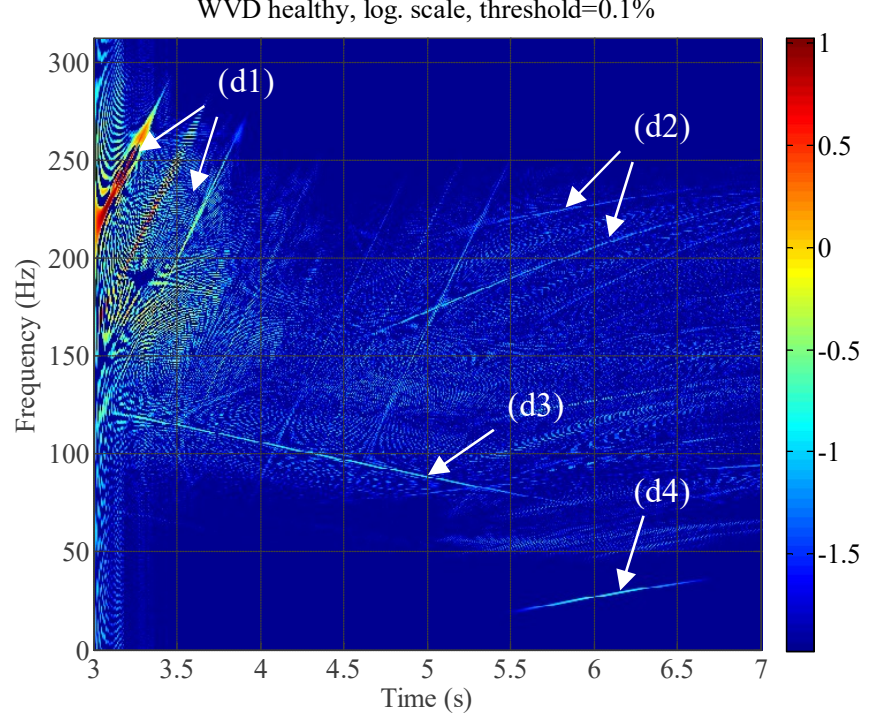

Fig. 9. High order rotor asymmetry harmonics in a healthy motor.

\section{Detection of rotor asymmetries}

If no band pass filter is used, the LSH 50 caused by such light rotor cage constructive asymmetry prevails during the last part of the time-frequency distribution, as seen in Fig. 10, where the PSH-1 (e1) and the LSH+50 (e2) feature similar energies.

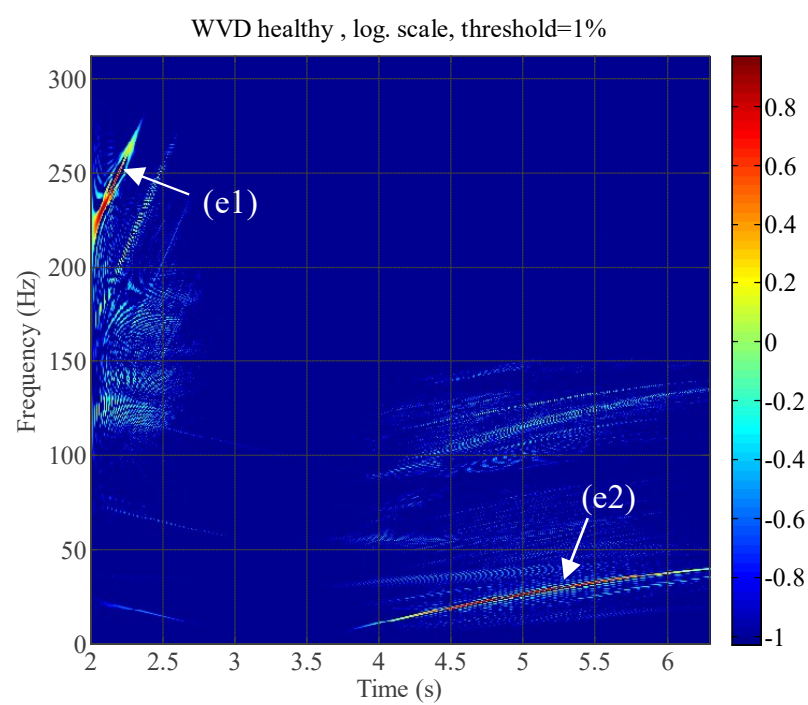

Fig. 10. WVD of a healthy motor. Wideband analysis. A PSH (e1) and the LSH 50 (e2) are detected.

Finally, the proposed approach is applied to the induction Motor I, having a broken bar cut at one of its ends. In this case (Fig. 11), the LSH+50 (f1) prevails, setting its magnitude the color scale and thus dimming the PSH-1 (f4). Further harmonics related to the rotor asymmetry are shown, such as a $\mathrm{LSH}+250 f(5-4 s)$ (f2) and a USH (f3) of the main current component.

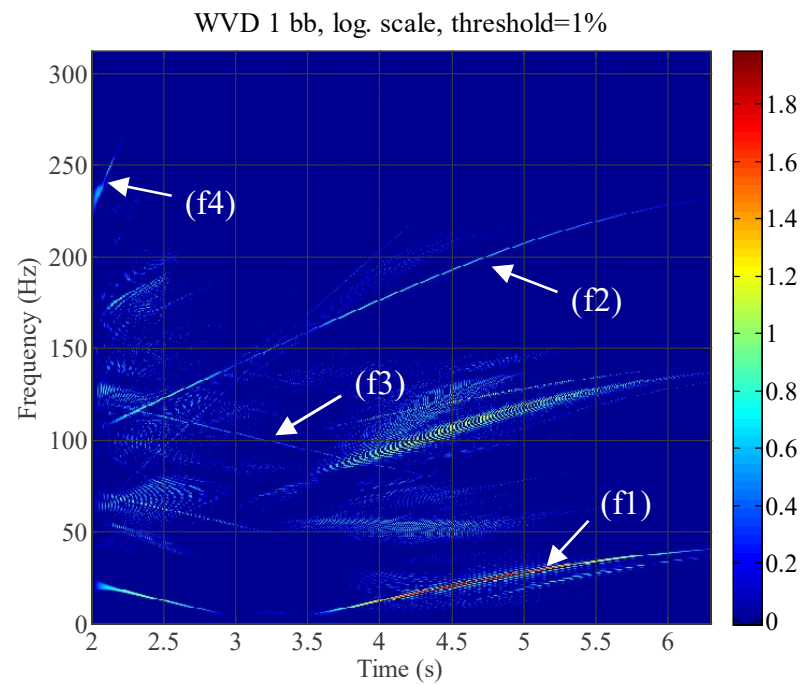

Fig. 11. WVD of a motor having one broken bar. Wideband analysis. Both high (f2, LSH 250) and low frequency harmonics (f1, LSH 50) related to the rotor asymmetry are detected. 
The use of advanced equiripple notch FIR filters allows the representation of the frequency evolving harmonics on a seamless time-frequency diagram that shows their detailed evolution, hence allowing its easy identification, despite the pretreatment procedure of eliminating the constant frequency harmonics. Furthermore, these FIR filters permit the representation in the same diagram of the evolution of both high and low frequency harmonics, enabling a full wideband analysis of a direct startup transient, which was not achieved using IIR filters in [18].

\section{Detection of eccentricities}

For the detection of eccentricities the phase current of a motor type II connected in delta is studied using equiripple filters, since the time-frequency box does not include the initial impulse. For this two pole pair machine the startup lasts only 3 seconds. Fig. 12 shows the WVD of a healthy machine centered in the third harmonic. The diagram has been widened to stationary operation up to second 5.

In this case, a PSH can be appreciated, as it evolves rapidly towards higher frequencies (g1). Also, a LSH 150 is detected (g2), as well as the EC 175 (g3) during stationary operation, the first related to a rotor asymmetry and the second to eccentricity. Both are caused by constructive tolerances. However, if the phase signal a Motor type II having 20\% static eccentricity and $50 \%$ dynamic eccentricity is examined, the characteristic sidebands around the main supply harmonic and its multiples appear, in especial, around the third multiple of the main harmonic for this delta connection.

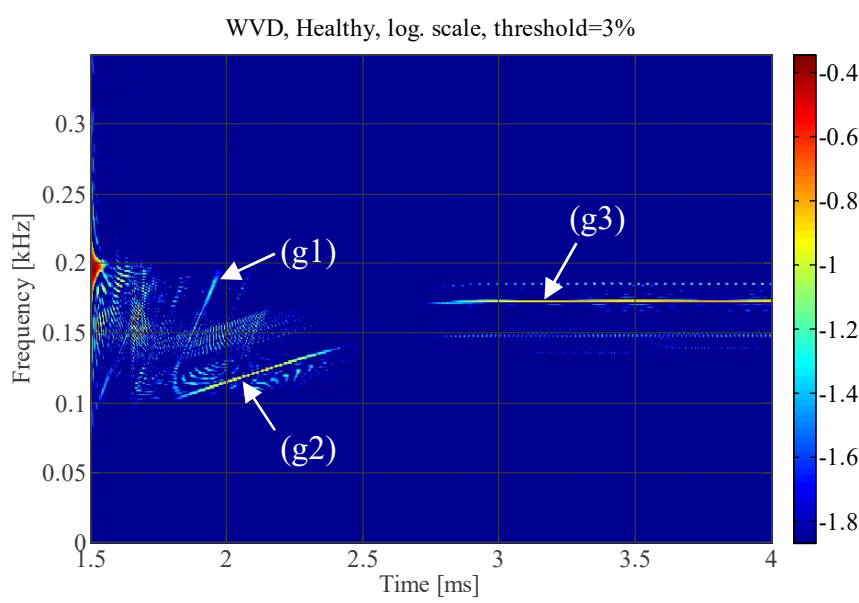

Fig. 12. WVD of the phase current of a healthy motor, second part of the startup and stationary operation. Analysis centered on the third harmonic. Rotor asymmetry (g2) and eccentricity harmonics (g3) related to constructive tolerances are detected.

Fig. 13 shows the Wigner-Ville Distribution of the startup (up to second 4) and stationary current, filtered by means of a FIR bandpass filter. The band studied spans, as in Fig. 13, from $120 \mathrm{~Hz}$ to $180 \mathrm{~Hz}$.

The Figure clearly presents the characteristic evolution of the EC-125 (h1) and EC+175 (h2) eccentricity components, which enables an easy identification of the fault.

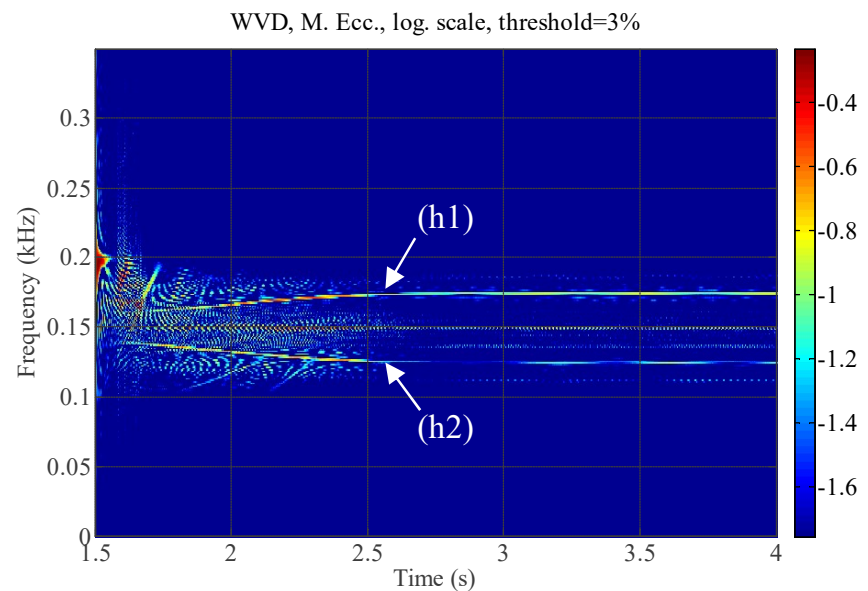

Fig. 13. WVD of a motor with mixed eccentricity. Analysis centered in the third supply harmonic. Both lower and upper sidebands related to the eccentricity are shown $(\mathrm{h} 1, \mathrm{~h} 2)$.

\section{E. Quantification}

For the quantification purpose, the procedure exposed in Section III is applied; firstly selecting, using low and high band pass FIR filters, a time-frequency box in which one fault harmonic prevails and then computing the instantaneous frequency in that box by (15). The smoothed result accurately tracks this harmonic and thus enables the quantification of its energy by integrating the result of the WVD on a band centered on its evolution.

Fig. 14 shows one of these time-frequency boxes, focused on the second part of a direct startup, low frequencies, for a motor having a broken bar, that is, it presents in 3 dimensions the area whose limits are between 3.5 and 6 seconds and 0 and $45 \mathrm{~Hz}$ in Fig. 11. In such conditions, the WVD works in its optimal conditions, since a monotonic chirp causes little cross terms, displaying very accurately the evolution (path) and energy (height) of the LSH 50 for a motor having one broken bar. Fig 15 depicts the smoothed instantaneous frequency, that is, the trace on the time -frequency diagram used for defining a dynamic band in which the energy has been integrated, for both the broken bar case presented in Fig. 14, and for a healthy motor. The lengthening of the startup period due to small differences in motor temperature does not hinder the correct tracking of the LSH even without the use of a speed sensor.

Finally, Fig. 16 shows the detected energy on a $4 \mathrm{~Hz}$-wide strip centered on the instantaneous frequency shown in Fig. 15, that is, the outline of the crest shown in Fig. 14 but in this case, for a healthy machine. The shape of the energy distribution remains the same compared to Fig. 14, but the values are an order of magnitude lower. 


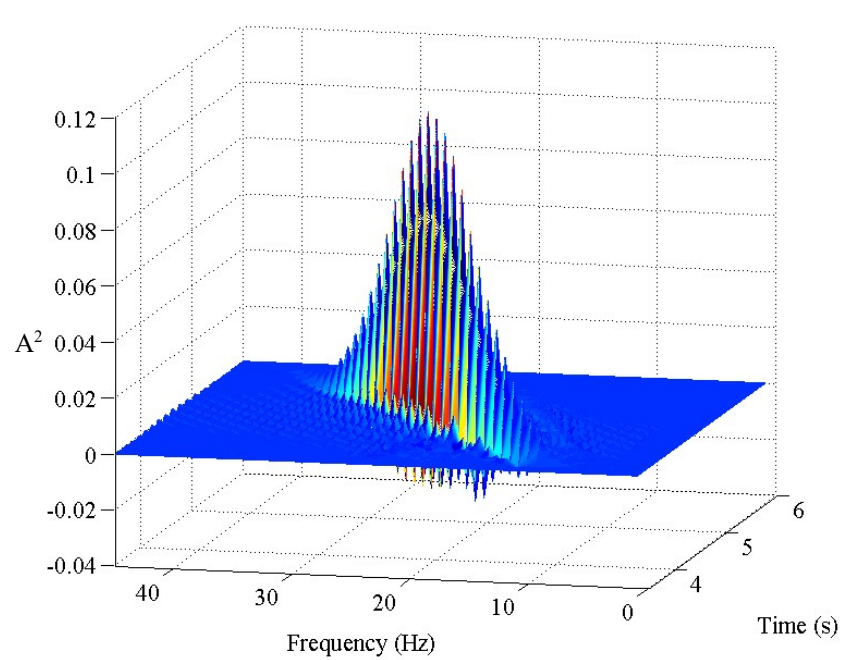

Fig. 14. DWV of the low sideband harmonic (LSH 50) evolving during the last part of the startup for a machine having one broken bar.

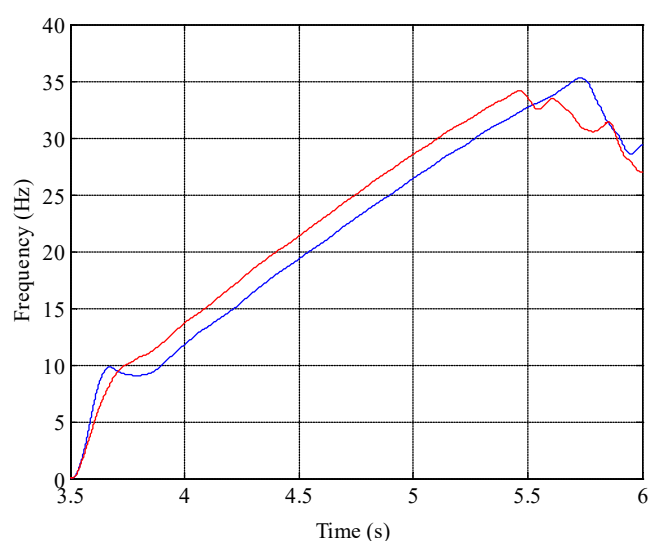

Fig. 15. Smoothed instantaneous frequency of the LSH 50: blue, healthy machine; red, machine with a broken rotor bar

In addition to the LSH, it is advisable to study the effect of the rotor asymmetry in higher order harmonics [19]. With this aim, a time-frequency box is isolated by means of notch and band pass filters between times 3 and 7 seconds and frequencies 150 and $250 \mathrm{~Hz}$, to track the main $\mathrm{LSH}+250$, that is, the $f(5-4 s)$ harmonic, which would indicate a double fault separated by a half pole pitch. The value of the quantification indicators obtained for the rotor asymmetry, are shown in Table V.

It must be noted that the variation of the parameter $\gamma_{\mathrm{W}}$ is similar but greater than the variation of the amplitudes of the same harmonics obtained studying the FFT spectrum during stationary operation of the motor. Therefore, the proposed method is more precise, as it corresponds to the heavy conditions the motor undergoes in a direct startup.

Moreover, for the case of the quantification of the eccentricity, other two time-frequency boxes have been correspondingly selected. The time range for both of them spans between seconds 2 and 3 whilst their frequency limits are between 120 and $140 \mathrm{~Hz}$ and 160 and $180 \mathrm{~Hz}$. The results are presented in Table V. There are no values for the healthy case since no eccentricity harmonic appears during the startup.

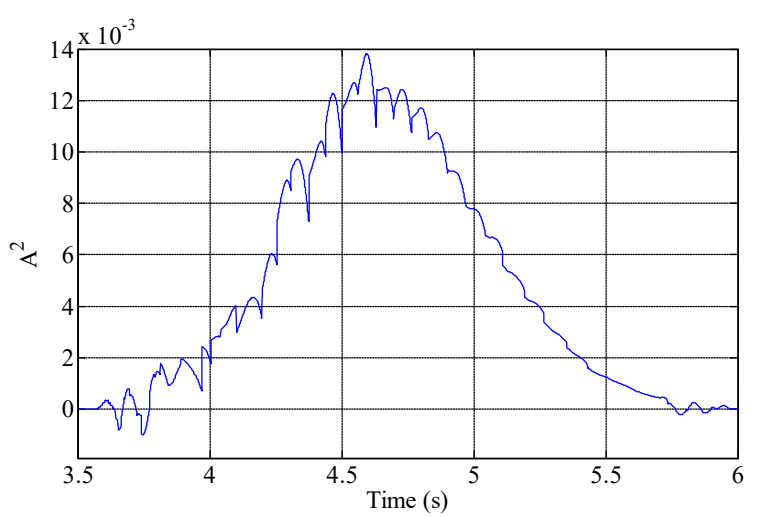

Fig. 16 Computed energy density in a $4 \mathrm{~Hz}$ width band centered on the trace of the instantaneous frequency shown in Fig. 15, blue line (healthy motor). The energy level can be compared to Fig. 14 for a motor featuring a broken bar.

TABLE V

QUANTIFICATION RESULTS

\begin{tabular}{cccc}
\hline State & $\begin{array}{c}\text { Motor } \\
\text { type }\end{array}$ & Harmonic & $\gamma_{\mathbf{W}}(\mathbf{d B})$ \\
\hline \multirow{3}{*}{ Healthy } & I & LSH 50 & 42.6 \\
& I & LSH 250 & 63.3 \\
& II & EC 125 & - \\
1 broken bar & II & EC 175 & - \\
Mixed & I & LSH 50 & 31.9 \\
Eccentricity & I & LSH 250 & 43.2 \\
\hline
\end{tabular}

Fig. 17 shows the smoothed energy detected. Up to second 3 , it corresponds to the startup (j1), in which the energy of both sidebands reaches twice the level of stationary operation $(j 2, j 3)$.

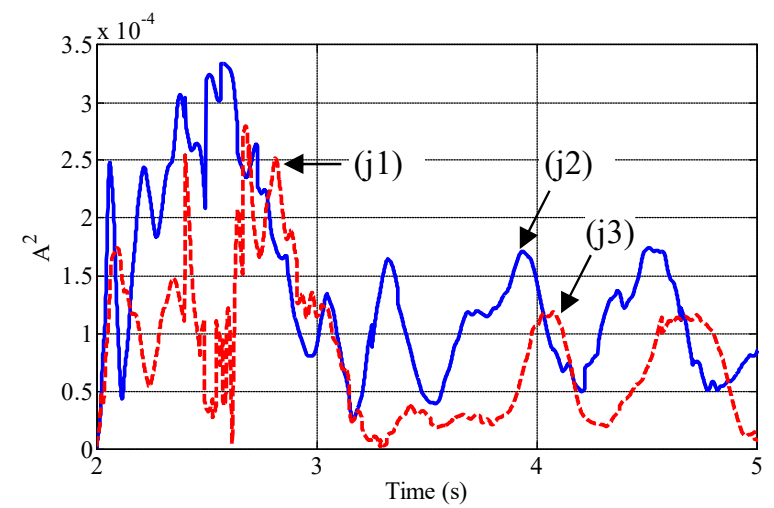

Fig. 17. Smoothed computed energy density in a $4 \mathrm{~Hz}$ width band centered on the trace of the instantaneous frequency for EC 175, blue line, and EC 125, red line.

\section{CONCLUSIONS}

This paper demonstrates the validity of a new method combining advanced notch FIR filters and the Wigner-Ville distribution in order to provide seamless high resolution timefrequency diagrams whose great amount of information allows performing the diagnosis of rotor asymmetries and eccentricities in induction machines directly connected to the grid even in the most difficult cases.

Unlike other approaches, the procedure increases its 
reliability by the simultaneous transient tracing of both high and low frequency fault-related harmonics whilst minimizing the cross-terms.

The method is completed by a robust tracking and quantification procedure for these frequency variable harmonics, which allows to precisely monitoring the evolution of the state of induction motors.

\section{APPENDIX}

The characteristics of the motors tested are:

Motor type I: Star connected, rated voltage $\left(\mathrm{U}_{\mathrm{n}}\right)$ : $400 \mathrm{~V}$, rated power $\left(\mathrm{P}_{\mathrm{n}}\right)$ : $1.5 \mathrm{~kW}, 1$ pole pairs, stator rated current $\left(\mathrm{I}_{1 \mathrm{n}}\right): 3.25$ A rated speed $\left(\mathrm{n}_{\mathrm{n}}\right): 2860 \mathrm{rpm}$.

Motor type II: Star connected, rated voltage $\left(\mathrm{U}_{\mathrm{n}}\right)$ : $400 \mathrm{~V}$, rated power $\left(\mathrm{P}_{\mathrm{n}}\right)$ : $1.1 \mathrm{~kW}, 2$ pole pairs, stator rated current $\left(\mathrm{I}_{1 \mathrm{n}}\right): 2.7 \mathrm{~A}$, rated speed $\left(\mathrm{n}_{\mathrm{n}}\right): 1410 \mathrm{rpm}$.

\section{REFERENCES}

[1] G.B Kliman, J Stein, and R.D. Endicott, "Noninvasive Detection of Broken Rotor Bars in Operating Induction Motors," IEEE Trans. on Energy Convers., vol. 3, no. 4, December 1988, pp. 873-879

[2] R Cameron et W.T. Thomson and A.B. Dow, "Vibration and current monitoring for detecting airgap eccentricity in large induction motors," IEE Proceedings, vol. 133, pt. B, no. 3, May 1986, pp. 155-163.

[3] S. H. Kia, H. Henao, G. -A. Capolino, "Windings monitoring of wound rotor induction machines under fluctuating load conditions", IECON 2011 - 37th Annual Conference on IEEE Industrial Electronics Society, 2011 , pp: $3459-3465$

4] J Cusidó, L. Romeral, J.A. Ortega, A. Garcia, J.R. Riba, "Wavelet an PDD as fault detection techniques", Electric Power Systems Research, Vol. 80, no.8, pp. 915-924, August 2010

[5] S. Nandi, S. Ahmed, H. A. Toliyat, "Detection of Rotor Slot and Other Eccentricity Related Harmonics in a Three Phase Induction Motor with Different Rotor Cages", IEEE Trans. on Energy Convers., vol. 16, no. 3, September 2001.

[6] S.H. Kia, H. Henao and G.A. Capolino, "Diagnosis of Broken Bar Fault in Induction Machines Using Discrete Wavelet Transform without Slip Estimation", Conference Record of the 2007 IEEE Industry Applications Conference, 2007. 42nd IAS Annual Meeting, 2007 pp: 1917 - 1922.

[7] J. Pons-Llinares, J. A. Antonino-Daviu, M. Riera-Guasp, M. PinedaSanchez, and V. Climente-Alarcon, "Induction motor diagnosis via frequency B-splines," IEEE Trans. Ind. Electron., vol. 58, no. 5, May 2011, pp. 1530-1544

[8] A. Ordaz-Moreno, R. Romero-Troncoso, J.A. Vite-Frías, J. RivieraGillen, A.García-Pérez, "Automatic online diagnostic algorithm for broken-bar detection on induction motors based on Discrete Wavelet Transform for FPGA implementation", IEEE Trans. Ind. Electron., Vol. 55, no.5, May 2008, pp.2193-2200

[9] J.A. Antonino-Daviu, M. Riera-Guasp, M. Pineda-Sanchez and R.B. Perez, "A critical comparison between DWT and Hilbert-Huang-based methods for the diagnosis of rotor bar failures in induction machines" IEEE Trans. Ind. Applic., Vol. 45, no. 5, 2009, pp. 1794-1803

[10] R. Burnett, J. F. Watson, S. Elder, "The application of modern signal processing techniques for use in rotor fault detection and location within three-phase induction motors," Signal Processing, vol. 49, No. 1, pp. 5770, February 1996

[11] Rajagopalan, S.; Aller, J.M.; Restrepo, J.A.; Habetler, T.G.; Harley, R.G., Detection of Rotor Faults in Brushless DC Motors Operating Under Nonstationary Conditions, Industry Applications, IEEE Transactions on, Vol. 42, no. 6, 2006, pp. 1464-1477

[12] Rajagopalan, J.M.; Restrepo, S.; Aller, , J.A.; Habetler, T.G.; Harley, R.G., Nonstationary Motor Fault Detection Using Quadratic TimeFrequency Representations, Industry Applications, IEEE Transactions on, Vol. 44, no. 3, 2008, pp.735-744

[13] J. A. Rosero, L. Romeral, J. A. Ortega y E. Rosero, Short-Circuit Detection by Means of Empirical Mode Decomposition and WignerVille Distribution for PMSM Running Under Dynamic Condition, IEEE Trans. Ind. Electron., vol. 56, no. 11, 2009, pp. 4534-4547
[14] J. Urresty, J. Riba, J. Ortega y J. Cárdenas, «Stator Short Circuits in PMSM by Means of the Zhao-Atlas-Marks Distribution and Energy Calculation,» 13th European Conference on Power Electronics and Applications, EPE '09, 2009, pp. 1-9, 2009

[15] J. Rosero, J. Cusido, A. Garcia-Espinosa, J. A. Ortega y L. Romeral, «Broken Bearings Fault Detection for a Permanent Magnet Synchronous Motor under non-constant working conditions by means of a Joint Time Frequency Analysis,» de Industrial Electronics, 2007. ISIE 2007. IEEE International Symposium on, 2007

[16] M. Blödt, J. Regnier y J. Faucher, "Distinguishing Load Torque Oscillations and Eccentricity Faults in Induction Motors Using Stator Current Wigner Distributions," IEEE Trans. Industry Applications, vol. 45, no. 6, 2009, pp. 1991-2000, 2009

[17] V. Climente-Alarcon, J. A. Antonino-Daviu, M. Riera, R. PuchePanadero y L. A. Escobar, Wigner-Ville Distribution for the Detection of High Order Harmonics due to Rotor Asymmetries, IEEE International Symposium on Diagnostics for Electric Machines, Power Electronics and Drives, SDEMPED, Cargese, 2009.

[18] V. Climente-Alarcon, J. A. Antonino-Daviu, M. Riera-Guasp, R. PuchePanadero, L. Escobar, "Application of the Wigner-Ville Distribution for the Detection of Rotor Asymmetries and Eccentricity through HighOrder Harmonics", Electric Power Systems Research, no. 91, pp.28-36, October 2012

[19] M. Riera-Guasp, J. Pons-Llinares, F. Vedreno-Santos, J. A. AntoninoDaviu y M. Fernandez Cabanas, "Evaluation of the amplitudes of highorder fault related components in double bar faults", IEEE International Symposium on Diagnostics for Electric Machines, Power Electronics \& Drives (SDEMPED), 2011, Bologna, 2011

[20] V. Climente-Alarcon; M. Riera-Guasp; J. Antonino-Daviu; J. RogerFolch, F. Vedreno-Santos, "Diagnosis of rotor asymmetries in wound rotor induction generators operating under varying load conditions via the Wigner-Ville Distribution", Power Electronics, Electrical Drives, Automation and Motion (SPEEDAM), 2012 International Symposium on, Sorrento (Italy), 2012 , pp: 1378 - 1383

[21] H. Douglas, P. Pillay, and A. K. Ziarani, "Broken rotor bar detection in induction machines with transient operating speeds," IEEE Trans. Energy Convers., vol. 20, pp. 135-141, 2005.

[22] P. Zahradnik, M. Vlcek, "Fast analytical design algorithms for FIR notch filters", IEEE Transactions on Circuits and Systems I: Regular Papers, vol. 51, pp. $608-623$

[23] B. Ayhan, H. J. Trussell, M. Chow, and M. Song, "On the Use of a Lower Sampling Rate for Broken Rotor Bar Detection With DTFT and AR-Based Spectrum Methods," IEEE Trans. Ind. Electron., vol. 55, p. $1421,2008$.

[24] P. Zahradnik, B. Simak, M. Vlcek, "Fast and Robust Analytical Design of Equiripple Comb FIR Filters for Communication Purposes", ICN 2008. Seventh International Conference on Networking, 2008, pp. $588-$ 593.

[25] V. Climente-Alarcon, J. A. Antonino-Daviu, E. Strangas, M. RieraGuasp, "Bar Breakage Mechanism and Prognosis in an Induction Motor", Proc. of IEEE International Symposium on Diagnostics for Electric Machines, Power Electronics and Drives, SDEMPED, Valencia, 2013, pp. 538-545

[26] A. Bellini, F. Filippetti, G. Franceschini, C. Tassoni, G.B. Kliman, "Quantitative Evaluation of Induction Motor Broken Bars by Means of Electrical Signature Analysis“, IEEE Trans. Ind. Applic., Vol. 37, no. 5, September/October 2001, pp 1248-1255

[27] A. Khezzar, M.H. Kaikaa, M. K. Oumaamar, M. Boucherna, H.Razik, "On the use of slot harmonics as a potential indicator of rotor bar breakage in the induction machine", IEEE Trans. Ind. Electron., Vol. 56, No. 11, November 2009, pp 4592-4605.

[28] M. Sahraoui, A. Ghoggal, S. E. Zouzou, M. Benbouzid, "Dynamic Eccentricity in squirrel cage induction motors - Simulation and analytical study of its spectral signatures on stator currents", Simulation Modelling Practice and Theory, Vol. 16, No. 9, pp. 1503-1513, 2008.

[29] J. Faiz, B. M. Ebrahimi, B. Akin, H. A. Toliyat, "Dynamic analysis of mixed eccentricity signatures at various operating points and scrutiny of related indices for induction motors," Electric Power Applications, IET, vol. 4, no. 1, 2010, pp. $1-16$

[30] J. Antonino-Daviu, P. Jover Rodriguez, M. Riera-Guasp, M. PinedaSánchez, A. Arkkio, "Detection of Combined Faults in Induction Machines with Stator Parallel Branches through the DWT of the startup current" Mechanical Systems and Signal Processing, Elsevier, Vol. 23, No. 7, October 2009, pp 2336-2351 
[31] X. Chen and T. W. Parks, "Analytic design of optimal FIR narrow-band filters using Zolotarev polynomials," IEEE Trans. Circuits Syst., vol. CAS-33, pp. 1065-1071, Nov. 1986.

[32] M. Vlcek, R. Unbehauen, "Zolotarev Polynomials and Optimal FIR filters", IEEE Trans. Signal Proc. Vol. 47, No. 3, 1999, pp. 717-730

[33] L. Cohen, Time-Frequency Analysis. A.V. Oppenheim, Ed. Prentice Hall Signal Processing Series, New Jersey, 1995.

[34] F. Auger, P. Flandirn, P. Gonçalvès, O. Lemoine, "Time Frequency Toolbox to Use with Matlab" CNRS (France) \& Rice University (USA) 1995-1996

[35] M. Pineda-Sanchez, M. Riera-Guasp, J.A. Antonino-Daviu, J. RogerFolch, J. Perez-Cruz, R. Puche-Panadero, "Instantaneous Frequency of the Left Sideband Harmonic During the Start-Up Transient: A New Method for Diagnosis of Broken Bars" IEEE Trans. Ind. Electron., Vol. 56, no.11, November 2009, pp.4557-4570

[36] M. Riera-Guasp, J.A. Antonino-Daviu, M. Pineda-Sanchez, R. PuchePanadero, J. Perez-Cruz, "A general approach for the transient detection of slip-dependent fault components based on the discrete wavelet transform", IEEE Trans. Ind. Electron., Vol. 55, 2008, pp. 4167-4180

Vicente Climente-Alarcon (M'12) received his M.Sc. degrees in Chemical and Industrial Engineering in 2000 and 2011, and his Ph.D. degree in Electrical Engineering in 2012, all from the Universitat Politècnica de València (Spain).

He has worked as Assistant Professor in the School of Industrial Engineering of the mentioned university, on research tasks in the area of condition monitoring of electrical machines, and externally as a consultant in automation and management of power systems. Currently he is carrying out postdoctoral research at the Department of Electrical Engineering and Automation, Aalto University, Espoo, Finland.

Jose A. Antonino-Daviu (M'06-SM'12) received the M.Sc. degree in Electrical Engineering from the Universidad Politecnica de Valencia in 2000 and the Ph.D. degree in Electrical Engineering in 2006. He worked in the private sector, being involved in several international projects. Currently, he is Associate Professor in the School of Industrial Engineering of the mentioned university, where he develops his docent and research work. His primary research interests are condition monitoring of electric machines, wavelet theory and its application to fault diagnosis and design and optimization of electrical installations and systems.

Martín Riera-Guasp (M'04-SM'12) received the M. Sc. degree in industrial engineering and the Ph.D. degree in electrical engineering from the Universitat Politècnica de València, Valencia (Spain), in 1981 and 1987, respectively.

Currently, he is an Associate Professor with the Department of Electrical Engineering, Polytechnic University of Valencia. His research interests include condition monitoring of electrical machines, applications of the Wavelet Theory to electrical engineering and efficiency in electric power applications.

Miroslav Vlček was born in Prague, The Czech Republic, in 1951. He received the graduate degree int heoretical physics from Charles University, Prague, The Czech Republic, in 1974, and the Ph.D. degree in communication engineering and D.Sc. degree from the Czech Technical University (CTU), Prague, The Czech Republic, in 1979 and in 1994, respectively. From 1974 to 1993, he was with the Department of Circuit Theory, Faculty of Electric Engineering, CTU, where he is currently Professor of Theory of Electrical Engineering. Since 1995, he has additionally been the Head of the Department of Applied Mathematics, Faculty of Transportation Sciences, CTU. He was the Alexander-von-Humboldt Fellow at the University of Erlangen, Nürberg, Germany in 1988 and 1997 . He currently teaches courses in system theory and digital filter design. His scientific interests include filter design and digital signal processing, and theory of approximation and higher transcendental functions. 